\title{
Estradiol Pretreatment in an Ultrashort GnRH Combined with a GnRH Antagonist Protocol in A Cohort of Poor Responders Undergoing IVF/ICSI: A Case-control Study
}

\author{
CHARALAMPOS SIRISTATIDIS ${ }^{1}$, GEORGE SALAMALEKIS ${ }^{1}$, PARASKEVI VOGIATZI ${ }^{1}$, TEREZA VRANTZA ${ }^{1}$, \\ PARASKEVI MATSOTA ${ }^{2}$, VASILEIOS PERGIALIOTIS ${ }^{3}$ and NIKOLAOS PAPANTONIOU ${ }^{3}$ \\ ${ }^{1}$ Assisted Reproduction Unit, 3rd Department of Obstetrics and Gynecology, \\ Medical School, National and Kapodistrian University of Athens, Athens, Greece; \\ ${ }^{2} 2$ nd Department of Anesthesiology, Medical School, \\ National and Kapodistrian University of Athens, Athens, Greece; \\ ${ }^{3} 3$ rd Department of Obstetrics and Gynecology, Medical School, \\ National and Kapodistrian University of Athens, Athens, Greece
}

\begin{abstract}
Aim: To provide results of the use of estradiol pretreatment in a combination of an ultrashort gonadotropinreleasing hormone (GnRH) agonist and antagonist protocol, in an attempt to improve the clinical outcomes in "poor responders", according to the Bologna criteria, undergoing in vitro fertilization (IVF). Patients and Methods: We applied estradiol pretreatment to 20 participants before the initiation of a combination of an ultrashort GnRH agonist plus an GnRH antagonist protocol followed by high doses of gonadotropins; the control group consisted of 20 subfertile participants with matching age, body mass index (BMI), basal follicle-stimulating hormone (FSH) and anti-Müllerian hormone (AMH), antral follicle count ( $A F C$ ) and cause of subfertility, conforming with the same inclusion criteria and treated with a fixed GnRH antagonist protocol. The primary outcome measure was live birth, while the secondary outcomes included embryological and cycle parameters. Results: Live birth was determined in reduced rates in the study compared to the control group $(0 / 20$ $v s .3 / 20, p=0.231$ ) as also in the respective number of clinical pregnancies $(2 / 20 \mathrm{vs.} 5 / 20, p=0.407)$ and cancellations $(10 / 20$ vs. 6/20, $p=0.197)$, but none of these differences reached statistical significance. Also, most of the secondary parameters studied were similar for both groups. Conclusion: The study
\end{abstract}

Correspondence to: Charalampos Siristatidis, Assisted Reproduction Unit, 3rd Department of Obstetrics and Gynecology, Medical School, National and Kapodistrian University of Athens, Rimini 1,Chaidari, Athens 12642, Greece. Tel: +30 6932294994, email: harrysiri@yahoo.gr

Key Words: GnRH agonist, GnRH antagonist, estradiol pretreatment, poor responders, assisted reproductive technology. protocol does not seem to constitute an equally effective method compared to the GnRH antagonist protocol in the selected study population. The presented dissimilar results with those reported so far in the literature are possibly attributed to the small sample size and the strict criteria applied when labeling participants as "poor responders".

Several approaches in assisted reproduction technology (ART) require a higher yield of oocytes, in order to typically present with a greater number of embryos for each couple increasing their chances for a successful embryo transfer and a positive outcome, in terms of pregnancy. On this basis, controlled ovarian hyperstimulation $(\mathrm{COH})$ has been considered a routine approach in ART as it leads to a higher number of oocytes and embryos to transfer and, potentially, select from. However, advanced age of the female partner and the anticipated hormonal variations may lead to a reduced ovarian reserve and, thus, a weakened response to ovarian stimulation, minimizing the chances of retrieving the anticipated number of oocytes.

Poor ovarian response (POR) characterizes a significant proportion of ART patients and poses a serious challenge in assisted reproduction, due to the lowered response following external stimulation, the high cancellation rates observed in these cycles and the increased incidence of implantation failure that accompanies them as found to occur in $9-24 \%$ of these cycles (1). Ferraretti et al. have presented the Bologna criteria, according to which a poor responder in an ART cycle is identified in order to adjust the approach parameters to the degree of obtaining the best possible outcome for the subfertile women/couples. According to these criteria, poor responders are identified by (i) advanced maternal age or any other risk factors for POR; (ii) a previous POR and (iii) an abnormal ovarian reserve test (2). 
Various strategies have been employed over the years towards optimization in the management of POR and modified $\mathrm{COH}$ protocols have been implemented without concrete evidence on the compelling advantage for one protocol over another $(3,4)$. Some preliminary results demonstrated superiority of the microdose flare-up over the letrozole/antagonist (5), although both agonist and antagonist protocols have demonstrated similar clinical pregnancy and cycle cancellation rates (6). As in many other cases in ART, the most probable and efficient method so far is considered the individualization of the treatment protocols (7).

In an attempt to distinguish the optimal approach by means of stimulation protocol for these patients, the combined ultrashort GnRH agonist/GnRH antagonist (ultrashort GnRH-ag/GnRH-ant) protocol has been reported to result in a significantly higher number of follicles with a higher yield of oocytes retrieved and embryos transferred, as well as a reasonable clinical pregnancy rate in patients with previous failed IVF attempts and poor embryo quality (8). The rationale of employing this protocol for POR lies within the separate benefits that the accompanying adjuncts, prior to ovarian stimulation, may offer: Firstly, estradiol pretreatment prevents corpus luteum formation and the ensuing hormonal stimulation. The result lies on the reduction of the premature gradual exposure of follicles to follicle-stimulating hormone (FSH) in the late luteal phase. The final consequence is that during the subsequent early follicular phase of the next cycle, there is a considerable condensation of the mean follicular size together with a putative improvement in overall follicular size homogeneity (9-11). Thus, COH might act towards synchronization and progress of follicular development more effectively. Secondly, the short use of the GnRH agonist for 3 days facilitates the release of the $\mathrm{FSH}$ through the flare-up phenomenon to promote follicular development. Finally, the standardized use of the GnRH antagonist provides immediate luteinizing hormone ( $\mathrm{LH})$ suppression and prevention of ovulation of the generated oocytes by impeding a premature LH surge (12).

The purpose of this case-control study was to compare the efficacy of oestradiol pretreatment in a combined ultrashort GnRH-ag/GnRH-ant protocol to a conventional antagonist stimulation protocol in a cohort of poor responders undergoing in vitro fertilization-intracytoplasmic sperm injection (IVF-ICSI).

\section{Patients and Methods}

Patient population and study design. This prospective study was performed at the Assisted Reproductive Unit of the Third Department of Obstetrics and Gynecology, "Attikon" Hospital, Medical School, National and Kapodistrian University of Athens, Athens, Greece, from 2013 to August 2016. The initial design was a single center, prospective, randomized study (RCT) to be conducted to the referral University Hospital, registered at clinicalstudies.gov in November 2013 (NCT01798836) (13). The trial protocol was approved by the Scientific Board (protocol No 520/08-03-13) and Bioethics Committee of the Hospital (No of approval 3/08-03-13). Due to the low recruitment rate and the unfavorable results obtained in terms of live birth rates, the RCT was forced to a stop by the study coordinator. The relevant data are analyzed as a prospective case-control study in order to contribute the outcomes of the study, although in an altered nature.

Patients. The cohort of the study participants was 40 subfertile women undergoing $40 \mathrm{IVF} / \mathrm{ICSI}$ cycles, with either primary or secondary subfertility, labeled as "poor responders", according to the Bologna criteria. A written informed consent was obtained from all participants following consultation and before the initiation of the treatment cycles. After clinical evaluation along with previous medical and reproductive history, participants were categorized in terms of subfertility factor as follows: female (tubal infertility, ovulatory dysfunction and endometriosis), male, female and male factor and unexplained infertility.

The inclusion criteria for the study entry were: age $25-45$ years, body mass index (BMI) $\leq 35$ and $\geq 19$, normo-ovulatory patients (menstrual cycles ranging from 24 to 35 days) and basal FSH $\leq 15 \mathrm{mIU} / \mathrm{ml}$ (day 2 or 3 of menstrual cycle). Poor responders were identified through the Bologna criteria and complied with the respective denoted parameters (2). The exclusion criteria for the participation in the study were: increased basal hormonal levels of FSH at day 3 of the menstrual cycle ( $>15 \mathrm{IU} / \mathrm{l})$, increased BMI $>35$, history of autoimmune, endocrine or metabolic disorders, ovarian cystectomy or oophorectomy, severe endometriosis (stage III-IV) or severe male factor (azoospermia).

Twenty participants that met the inclusion criteria were enrolled in the study and treated with estradiol pretreatment and a combined GnRH ultrashort agonist-antagonist protocol. The control group consisted of 20 subfertile participants with matching age, BMI, basal FSH, antral follicle count (AFC), anti-Müllerian hormone $(\mathrm{AMH})$ and cause of subfertility, conforming with the same inclusion criteria and was treated with a GnRH antagonist protocol. Our analysis was performed to provide a direct comparison of the study and control groups.

Patients were enrolled during a 3-year study period and participated in the study only once. The number selected was based on previous reports (14-16).

Stimulation protocols and IVF. The study group received estradiol pretreatment and a combination of ultrashort GnRH agonist plus GnRH antagonist protocol, while the control group received a conventional GnRH antagonist protocol.

Estradiol pretreatment (Cyclacur ${ }^{\circledR}$ tabs, $2 \mathrm{mg}$, white only, Bayer Hellas, Athens, Greece) was administered at day 20 of the previous cycle and continued up to 10 days or stopped in the appearance of menses. For the ultrashort GnRH agonist protocol, $0.1 \mathrm{mg}$ triptorelin (Gonapeptyl ${ }^{\circledR}$, Ferring, Aalst, Belgium) or Arvekap ${ }^{\circledR}, 0.1 \mathrm{mg}$ (Ipsen, Ltd., Athens, Greece) was administered subcutaneously daily starting on day two of the menstrual cycle for three consecutive days. For the ovarian stimulation, recombinant FSH (r-FSH) in the form of either follitropin alpha $\left(\right.$ Gonal- $\mathrm{F}^{\circledR}$; Merck Serono Europe Ltd., Middlesex, UK) or follitropin beta (Puregon ${ }^{\circledR}$; MSD Ltd., Brussels, Belgium) was administered subcutaneously (s.c.) and initiated on day two of the cycle. A GnRH antagonist, either ganirelix (Orgalutran ${ }^{\circledR}$; MSD Ltd.) or cetrorelix (Cetrotide ${ }^{\circledR}$; Merck 
Serono Europe Ltd.) in fixed s.c. daily doses for 5 or 6 days were added, provided a 14-mm follicle was present on pelvic ultrasound evaluation and continued up to the day of human chorionic gonadotropin (hCG) administration.

For cycle monitoring, transvaginal ultrasonography was performed every $2 \sim 4$ days or as required. Starting doses were $300 \mathrm{IU}$ to $450 \mathrm{IU}$ and were adjusted individually according to FSH, AMH levels and previous response to IVF cycles of each participant, while further adjustments and monitoring frequency were dependent upon participants' response to the stimulation regime. When one or more follicles reached a diameter of $18 \mathrm{~mm}$, hCG ((10,000 IU Pregnyl ${ }^{\circledR}$ (MSD Ltd.) or $250 \mathrm{mg}$ Ovitrelle ${ }^{\circledR}$ (Merck Serono Europe Ltd.) was administered prior to transvaginal ultrasound-guided oocyte retrieval (OR) 36-38 hours later; the latter was performed under a standard protocol of general anesthesia, including intravenous administration of midazolam, propofol and fentanyl. Intracytoplasmic sperm injection (ICSI) was performed in male factor and in cases with low fertilization rate in previous cycles. Depending on the embryo quality, embryo transfer was performed either two or three days after the OR, whereas the maximum number of embryos transferred was two.

Luteal phase support was achieved by transvaginal administration of micronized progesterone either in the form of vaginal suppositories (Utrogestan ${ }^{\circledR}$; vaginal tablets, $200 \mathrm{mg}$ t.i.d; Basins Iscovesco, Paris, France) or gel (Vasclor ${ }^{\circledR}$; vaginal gel 8\%; Verisfield Ltd., London, UK) starting from the afternoon of the day of OR.

Outcome measures and variables' presentation. Our main outcome measure was live birth. Secondary outcome measures were total dose of gonadotropins used, endometrial thickness and number of follicles of 14-15 mm, 15-16 mm and $18 \mathrm{~mm}$ at day of hCG triggering, number of total and mature oocytes retrieved (MII) and fertilized, number of cleaved and transferred embryos, day of transfer, positive pregnancy test, cycle cancellation, clinical pregnancy and miscarriage rates.

Live birth was defined by the birth of a live fetus after 20 weeks of gestation and clinical pregnancy as the confirmed presence of an intrauterine sac with fetal heart activity through transvaginal ultrasound scan at seven weeks of gestation, following a positive serum $\beta$-hCG test. Cycle cancellation was forced in cases where premature ovulation was evident by the loss of follicle(s) prior to OR and in cases where there were no oocytes retrieved or no embryos produced for transfer or in failed fertilization. Miscarriage rate was defined as pregnancy loss earlier than 20 weeks of gestation, following a positive serum assay for pregnancy. Embryo quality for endometrial transfer or cryopreservation was assessed according to morphological criteria based on the overall blastomere number, size, appearance and degree of fragmentation (17).

Statistical analysis. Data are expressed as median (interquartile range). The normality of the distributions was assessed with Kolmogorov-Smirnov's test and graphical methods. We used the Mann-Whitney non-parametric test for the comparison of continuous variables due to their abnormal distribution. Categorical variables were assessed with the Chi-square test. Fisher's exact test was performed wherever the number of cases was $<5$. All tests were two-sided. Differences were considered as statistically significant if the null hypothesis could be rejected with $>95 \%$ confidence $(p<0.05)$. All tests were performed with the SPSS 21.0 package (IBM Corp. Released 2012; IBM SPSS Statistics for Windows, Version 21.0; Armonk, NY, USA).

\section{Results}

Overall, 40 women participated in the study, labeled as "poor responders", according to the Bologna criteria. Among them, 20 women were treated with the GnRH antagonist protocol, comprising the control group, while 20 women received estradiol pretreatment and the combination of the $\mathrm{GnRH}$ agonist and antagonist, comprising the study group. In this patient sample recruited in a timeline of more than three years, there were no dropouts after the initial enrollment.

Patients' and basic cycle characteristics are presented in Table I. Both groups were matched in terms of age, BMI, basal FSH, AFC and AMH values, duration and cause of subfertility, smoking habits, as well as parity (all $p$-values $>0.05$ ). The summary of outcomes is presented in Table II.

The number of oocytes retrieved was similar between groups (1 (0-8) vs. $2(0-11), p=0.341)$. The total dose of gonadotropins used for ovarian stimulation was similar between groups $(3,600 \mathrm{IU}(1,800-5,850)$ vs. 3,712 IU $(2,400$ $6,300), p=0.862)$. Similarly, at the day of hCG triggering, both number of follicles (irrespective of their size) and endometrial thickness did not differ; concurrently, the number of MII and fertilized oocytes and cleaved embryos was comparable between groups (all $p$-values $>0.05$ ). Also, day and number of embryo transfers were similar.

The number of cancelled cycles was higher in the study compared to the control group; however, this difference did not reach statistical significance $(p=0.197)$. Similarly, biochemical, clinical pregnancies and live births were lower in the study compared to the control group, but none of the differences reached significance (all $p$-values $>0.05$ ).

\section{Discussion}

In this prospective case-control study, we evaluated the efficacy of estradiol pretreatment in combination of an ultrashort GnRH agonist/GnRH antagonist protocol compared with a conventional GnRH antagonist protocol in 40 subfertile women undergoing IVF, labeled as "poor responders", according to the Bologna criteria. The outcomes examined included parameters of the stimulation processes in preparation for ART, as well as the embryological and clinical outcomes following the induced cycle. Our results demonstrated that live birth, comprising the primary outcome of the current study, was inferior in the study as compared to the control group but did not manage to reach statistical significance, similarly with cancellation, positive hCG and clinical pregnancy rates. We acknowledge the fact that the reduced cohort along with the increased cancellation rate reduces the value of the clinical results. 
Table I. Baseline and demographic characteristics of the participants.

\begin{tabular}{lccc}
\hline & Study group $(\mathrm{n}=20)$ & Control group & $p$-Value $(\mathrm{n}=20)$ \\
\hline Age (years) & $41.5(34.0-45.0)$ & $42.5(36.0-45.0)$ & 0.738 \\
BMI $\left(\mathrm{kg} / \mathrm{m}^{2}\right)$ & $24.5(20.1-34.0)$ & $23.9(18.0-35.0)$ & 0.211 \\
Smoking & $8 / 20$ & $9 / 20$ & $>0.999$ \\
Nulliparity & $18 / 20$ & $16 / 20$ & 0.661 \\
Primary subfertility & $13 / 20$ & $11 / 20$ & 0.519 \\
Cause of subfertility & & & \\
$\quad$ Unexplained & $4 / 20$ & $6 / 20$ & 0.465 \\
Male & $4 / 20$ & $7 / 20$ & 0.288 \\
Ovulatory & $4 / 20$ & $3 / 20$ & $>0.999$ \\
Tubal & $7 / 20$ & $6 / / 20$ & 0.736 \\
$\quad$ Endometriosis & $0 / 20$ & $0 / 20$ & $\mathrm{~N} / \mathrm{A}$ \\
Duration of subfertility (years) & $2.8(1.0-8.0)$ & 0.547 \\
AFC & $3(1-11)$ & $3(1.0-8.0)$ & 0.221 \\
Basal FSH (IU/l) & $9.7(4.7-15.0)$ & $9.9(4.0-15.0)$ & 0.841 \\
AMH (ng/ml) & $1.2(0-4.9)$ & $1.8(0-6.7)$ & 0.341 \\
\hline
\end{tabular}

Numeric data are presented as medians ( 5 and $95 \%$ confidence interval). Percentages are presented as absolute numbers (5 and $95 \%$ confidence interval). $p<0.05$ was considered statistically significant. AFC, Antral follicle count; AMH, anti-Müllerian hormone; BMI, body mass index; FSH, follicle-stimulating hormone.

Table II. Characteristics of the stimulation cycle and clinical outcomes of the two studied groups.

\begin{tabular}{lccc}
\hline & Study group $(\mathrm{n}=20)$ & Control group (n=20) & $p$-Value \\
\hline Dose of gonadotropins (IU) & $3712(2400-6300)$ & $3600(1800-5850)$ & 0.862 \\
Number of cancelled cycles & $10 / 20$ & $6 / 20$ & 0.197 \\
Endometrial thickness (mm) & $8.3(3.3-10.2)$ & $7.9(4.8-10.2)$ & 0.253 \\
Number of follicles 14-15 mm & $0(0-5)$ & $0.5(0-2)$ & 0.224 \\
Number of follicles 16-17 mm & $0(0-2)$ & $1(0-5)$ & 0.925 \\
Number of follicles 18 mm & $1(0-4)$ & $1(0-5)$ & 0.718 \\
Number of oocytes retrieved & $1(0-8)$ & $2(0-11)$ & 0.341 \\
Number of M II oocytes & $1(0-4)$ & $1(0-7)$ & 0.211 \\
Number of fertilized oocytes & $1(0-4)$ & $1(0-7)$ & 0.445 \\
Number of cleaved embryos & $1(0-3)$ & $1(0-7)$ & 0.445 \\
Number of transferred embryos & $1(0-2)$ & $1(0-2)$ & 0.398 \\
Day of endometrial transfers & & & \\
$\quad$ Second day & $2 / 20$ & $4 / 20$ & 0.441 \\
Third day & $8 / 20$ & $10 / 20$ & 0.407 \\
Positive hCG & $2 / 20$ & $5 / 20$ & 0.407 \\
Clinical pregnancy & $2 / 20$ & $5 / 20$ & 0.999 \\
Miscarriage & $2 / 20$ & $2 / 20$ & 0.231 \\
Live births & $0 / 20$ & $3 / 20$ & \\
\hline
\end{tabular}

Numeric data are presented as medians ( 5 and $95 \%$ confidence interval). $p<0.05$ was considered statistically significant. hCG, human chorionic gonadotropin.

There is still controversy over the optimal protocol to be offered in poor responders and this still remains an existing challenge, with fertility experts struggling to find the optimal solution to offer, before suggesting oocyte donation at several occasions. The most common protocols for this kind of management are the microdose flare-up and antagonist protocols (20), whereas there is insufficient evidence to support the routine use of any particular intervention (3). The reason is probably linked with the fact that poor responders are not a homogeneous group of women with regards to pregnancy prospects, while female age and number of oocytes retrieved might modulate the chances for pregnancy (21). Current notion suggests that individualization of treatment strategies through the selective use of $\mathrm{GnRH}$ 
analogues and the fine-tuning of the gonadotropin dose on the basis of potential ovarian response, could be the norm. In our study, we have strictly applied the Bologna criteria developed by the European Society for Human Reproduction and Embryology consensus in 2011 (2): this was quite different to the existing reports, which have applied the same protocol. Correspondingly, as stated in a recent systematic review, there were 41 different definitions, among 47 randomized trials, employed for the patients demonstrating poor ovarian response (4). The current study was initially designed as an RCT but the low outcome rates obtained following a prescheduled interim analysis were the main grounds for discontinuing the recruitment and present the results as a case-control study, using a matched group, in terms of baseline and demographic characteristics, as control.

Concerning the therapeutic protocol, a current systematic review encompassing eight (mainly retrospective cohort) studies showed that, although there was no clear conclusion concerning live birth rates, both cancellation and clinical pregnancy rates favored estradiol pretreatment in a $\mathrm{GnRH}$ antagonist protocol, compared to the non-use of estradiol in poor responders (22). Similarly, earlier conducted studies reported higher pregnancy rates (around 14\%) compared to the previous cycles in 21 cases (8) or improvement in clinical pregnancy rates $(50 \%)$, when compared with the previous cycles of those patients (14). In our study, neither estrogen pretreatment nor the combination of a GnRH ultrashort and a GnRH antagonist protocol were able to show any beneficial effect on the cycle outcomes, when compared to a conventional GnRH antagonist protocol; that was probably due to the high cancellation rates, leading to inferior both clinical and live birth rates. In the reported cases, estradiol pretreatment did not manage to lead to the recruitment of sufficient number of antral follicles, leading to a very low number of oocytes retrieved, matured and fertilized, coupled with a high cancellation rate, which reached a low percentage $(30 \%)$. It seems that in true poor responders undergoing IVF, the low number of oocytes retrieved comprises the first and one of the main parameters of their failure to achieve favorable results with any protocols used to date. We believe that there are two main reasons that we found different results from those of the current literature. Firstly, the number of participants was low not permitting firm conclusions to be drawn. We also feel that if the recruitment continued, a significant trend would have been observed favoring the GnRH antagonist regimen. We stopped the recruitment, as it would be unethical to continue offering a treatment modality to this category of patients that resulted in zero live birth rates. Secondly, the recruitment of participants was done strictly following the Bologna criteria; of note, the wide difference in defining "poor response" in ART is one of the main etiologies for the discrepancy of the results currently published (4).
A definite limitation of the current study is the lack of randomization and the reduced cohort size. We fully acknowledge the difficulty of performing well-powered RCTs to answer the particular clinical question. In this context, a robust answer should emerge if a large scale RCT was to be conducted. Still, evidence remains poor in this field. On the other hand, until such data are available, clinical guidance has to be influenced from studies with a lower scientific power in the hierarchy of evidence.

In conclusion, estradiol pretreatment followed by the combination of both GnRH agonist and antagonist protocols does not seem to constitute an equally effective method as compared to a standard GnRH antagonist regimen to be offered in subfertile women characterized as "poor responders" seeking ART. The nature of the study does not permit us to draw firm conclusions. We could not verify the results of previous reports where estradiol pretreatment appeared effective in $\mathrm{COH}$ protocols for this group of subfertile patients. The treatment of these patients still remains a challenge and, on these terms, the strict criteria characterizing this group and the nature of the condition itself hinders the recruitment of a large cohort, along with the anticipated increased cancellation rate that reduces the clinical value of the evidence provided. Clinical experience, individualization and case-oriented evaluation of these patients still represents the most attractive option to offer as Bologna criteria introduce an evaluation standard to determine poor responders.

\section{Conflicts of Interest}

All Authors declare no conflict of interest.

\section{Acknowledgements}

The Authors wish to thank the clinical, paramedical and laboratory team of the Assisted Reproduction Units of the 3rd Department of Obstetrics and Gynecology, "Attikon" Hospital in Athens, Greece. There was no finding for the current work.

\section{References}

1 Fasouliotis S, Simon A and Laufer N: Evaluation and treatment of low responders in assisted reproductive technology: A challenge to meet. J Assist Reprod Genet 17: 357-373, 2000.

2 Ferraretti AP, La Marca A, Fauser BC, Tarlatzis B, Nargund $\mathrm{G}$ and Gianaroli L: ESHRE working group on poor ovarian response definition. ESHRE consensus on the definition of 'poor response' to ovarian stimulation for in vitro fertilization: the Bologna criteria. Hum Reprod 26: 16161624, 2011.

3 Pandian Z1, McTavish AR, Aucott L, Hamilton MP and Bhattacharya S: Interventions for 'poor responders' to controlled ovarian hyper stimulation (COH) in in vitro fertilisation (IVF). Cochrane Database Syst Rev 20(1): CD004379, 2010. 
4 Polyzos NP and Devroey P: A systematic review of randomized trials for the treatment of poor ovarian responders: Is there any light at the end of the tunnel? Fertil Steril 96: 1058-1061.e7, 2011.

5 Song Y, Li Z, Wu X, Wang X, Xiao J and Wang B: Effectiveness of the antagonist/letrozole protocol for treating poor responders undergoing in vitro fertilization/intracytoplasmic sperm injection: A systematic review and meta-analysis. Gynecol Endocrinol 30: 330-334, 2014.

6 Xiao J, Chang S and Chen S: The effectiveness of gonadotropinreleasing hormone antagonist in poor ovarian responders undergoing in vitro fertilization: A systematic review and metaanalysis. Fertil Steril 100: 1594-1601.e1-9, 2013.

7 La Marca A and Sunkara SK: Individualization of controlled ovarian stimulation in IVF using ovarian reserve markers: From theory to practice. Hum Reprod Update 20: 124-140, 2014.

8 Orvieto R, Kruchkovich J, Rabinson J, Zohav E, Anteby EY and Meltcer S: Ultrashort gonadotropin-releasing hormone agonist combined with flexible multidose gonadotropin-releasing hormone antagonist for poor responders in in vitro fertilization/ embryo transfer programs. Fertil Steril 90: 228-230, 2008.

9 Fanchin R, Cunha-Filho JS, Schonäuer LM, Kadoch IJ, CohenBacri P and Frydman R: Coordination of early antral follicles by luteal estradiol administration provides a basis for alternative controlled ovarian hyperstimulation regimens. Fertil Steril 79: 316-321, 2003.

10 Fanchin R, Salomon L, Castelo-Branco A, Olivennes F, Frydman $\mathrm{N}$ and Frydman R: Luteal estradiol pre-treatment coordinates follicular growth during controlled ovarian hyperstimulation with GnRH antagonists. Hum Reprod 18: 2698-2703, 2003.

11 Fanchin R, Schönauer L, Cunha Filho J, Kadoch I, Cohen-Bacrie $\mathrm{P}$ and Frydman R: Luteal E2 administration reduces size and improves homogeneity of selectable follicles on cycle-day 3 : Bases for novel controlled ovarian hyperstimulation $(\mathrm{COH})$ concepts. Fertil Steril 76: S90, 2001.

12 Kolibianakis EM, Zikopoulos K, Schiettecatte J, Smitz J, Tournaye H, Camus M, Van Steirteghem AC and Devroey P: Profound LH suppression after GnRH antagonist administration is associated with a significantly higher ongoing pregnancy rate in IVF. Hum Reprod 19: 2490-2496, 2004.

13 Siristatidis C: Oestradiol pre-treatment in an ultrashort flare GnRH agonist/GnRH antagonist protocol in poor responders undergoing IVF. Clinicaltrials gov NCT01798836, 2013 (https://clinicaltrials.gov/ct2/show/NCT01798836)
14 Orvieto R, Nahum R, Rabinson J, Gemer O, Anteby EY and Meltcer S: Ultrashort flare GnRH agonist combined with flexible multidose GnRH antagonist for patients with repeated IVF failures and poor embryo quality. Fertil Steril 91: 1398-1400, 2009.

15 DiLuigi AJ, Engmann L, Schmidt DW, Benadiva CA and Nulsen JC. A randomized trial of microdose leuprolide acetate protocol versus luteal phase ganirelix protocol in predicted poor responders. Fertil Steril 95: 2531-2533, 2011.

16 Ata B, Zeng X, Son WY, Holzer H and Tan SL. Follicular synchronization using transdermal estradiol patch and GnRH antagonists in the luteal phase; does it increase oocyte yield in poor responders to gonadotropin stimulation for in vitro fertilization (IVF)? A comparative study with microdose flareup protocol. Gynecol Endocrinol 27: 876-879, 2011.

17 Ziebe S, Petersen K, Lindenberg S, Andersen AG and Gabrielsen A, Andersen AN. Embryo morphology or cleavage stage: How to select the best embryos for transfer after in vitro fertilization. Hum Reprod 12: 1545-1549, 1997.

18 Facey KM and Lewis JA: The management of interim analyses in drug development. Stat Med 17: 1801-1809; 1811-1812, 1998.

19 Todd S, Whitehead A, Stallard N and Whitehead J: Interim analyses and sequential designs in phase III studies. Br J Clin Pharmacol 51: 394-399, 2001.

20 Eftekhar M, Mohammadian F, Yousefnejad F and Khani P: Microdose $\mathrm{GnRH}$ agonist flare-up versus ultrashort $\mathrm{GnRH}$ agonist combined with fixed gnrh antagonist in poor responders of assisted reproductive techniques cycles. Int J Fertil Steril 6: 266-271, 2013.

21 Oudendijk JF1, Yarde F, Eijkemans MJ, Broekmans FJ and Broer SL: The poor responder in IVF: is the prognosis always poor?: A systematic review. Hum Reprod Update 18: 1-11, 2012.

22 Reynolds KA, Omurtag KR, Jimenez PT, Rhee JS, Tuuli MG and Jungheim ES: Cycle cancellation and pregnancy after luteal estradiol priming in women defined as poor responders: A systematic review and meta-analysis. Hum Reprod 28: 29812989,2013
Received August 26, 2016

Revised September 7, 2016

Accepted September 8, 2016 